\title{
One-dimensional characters of excitons in carbon nanotubes
}

\author{
Seiji $\mathrm{Uryu}^{a}$ and Tsuneya Ando ${ }^{b}$ \\ ${ }^{a}$ Department of Materials Science and Engineering, Iwate University \\ 4-3-5 Ueda, Morioka, Iwate 020-8551, Japan \\ ${ }^{b}$ Department of Physics, Tokyo Institute of Technology \\ 2-12-1 Ookayama, Meguro-ku, Tokyo 152-8551, Japan
}

\begin{abstract}
Recent theoretical studies on exciton absorption in carbon nanotubes in an effective-mass approximation are reviewed. It is clarified that one-dimensional character plays important roles in optical properties of carbon nanotubes. For semiconducting tubes, exciton effects for polarization perpendicular to the tube axis cause prominent peaks in optical absorption spectra in spite of the depolarization effect. Calculated excited exciton energies for parallel polarization well reproduce measured energies for one- and two-photon absorption. Excitons can also exist in metallic carbon nanotubes.
\end{abstract}

Keywords: carbon nanotube, effective-mass approximation, exciton, optical absorption

\section{INTRODUCTION}

Carbon nanotubes are rolled up two-dimensional (2D) graphite sheets, which can be metallic or semiconducting depending on the chiral vector giving the circumference. Because of the quasi-one-dimensionality, exciton effects play dominant roles in their optical properties. In this paper, we review recent theoretical studies on exciton absorption in carbon nanotubes based on a unified scheme using an effective-mass-approximation. ${ }^{13}$

For light polarized parallel to the tube axis, it was predicted ${ }^{4,5}$ that the band gap is enhanced considerably by the Coulomb interaction and that exciton binding energy is comparable to but slightly smaller than this enhancement. As a result, the intensity of the optical absorption is focused on exciton energy levels lying higher than the band gap in the noninteracting case. This prediction was confirmed both theoretically ${ }^{6-10}$ and experimentally. ${ }^{11-13}$ For metallic nanotubes, the presence of exciton effects was theoretically suggested ${ }^{2,14,15}$ and also addressed experimentally. ${ }^{14}$

Excited excitons were also studied by two-photon absorption and other methods. ${ }^{3,16} 23$ In a one-particle model, the two-photon transition was prohibited between band edges. ${ }^{16}$ Photoluminescence experiments with two-photon absorption revealed that difference between the energies of one- and two-photon transitions is substantial, typically a few hundred meV, leading to the clear conclusion that the absorptions arise from excitons. ${ }^{20} 22$ Moreover, calculations with exciton effects were performed and used for the estimation of exciton binding-energy from experimental one- and two-photon peaks.

While for polarization perpendicular to the tube axis, it was shown based on a one-particle model that the depolarization effect considerably reduces absorption, leading to anisotropic optical properties. ${ }^{24,25}$ However, it was theoretically shown for semiconducting tubes that exciton effects also cause distinct peaks in absorption spectra though their intensity is reduced as compared to that for parallel polarization. ${ }^{1,7,9,26}$ Recent experiments observed absorption peaks with reduced intensity which are located at energies close to the second peaks for the parallel polarization. ${ }^{27}$ All of these are in good agreement with the theories.

In this paper, we show theoretical studies on exciton effects on cross-polarized-light absorption and twophoton absorption in semiconducting tubes and on excitons in metallic tubes. The paper is organized as follows: In Sec. 2 an effective-mass approximation is given. Numerical results are described in Sec. 3 and discussed in Sec. 4. Summary and conclusion are given in Sec. 5 . 


\section{EFFECTIVE-MASS APPROXIMATION}

\subsection{Energy bands and wave functions}

In a 2D graphite sheet shown in Fig. 1(a), the conduction and valence bands consisting of $\pi$ states cross at $K$ and $K^{\prime}$ points and the electron motion around these points is described well by a $\mathbf{k} \cdot \mathbf{p}$ equation corresponding to a relativistic Dirac equation with vanishing rest mass. Around the $K$ point, for example, it is given by 32

$$
\gamma(\vec{\sigma} \cdot \hat{\mathbf{k}}) \mathbf{F}(\mathbf{r})=\varepsilon \mathbf{F}(\mathbf{r}),
$$

where $\mathbf{F}(\mathbf{r})$ is a two-component envelope function, the $x$ and $y$ coordinates are chosen in the circumference and the axis direction, respectively, as shown in Fig. $1(\mathrm{~b}), \varepsilon$ is an eigen energy measured from the Fermi energy, $\gamma$ a band parameter, $\vec{\sigma}=\left(\sigma_{x}, \sigma_{y}\right)$ the Pauli spin matrix, and $\hat{\mathbf{k}} \equiv-i \vec{\nabla}$ a wave vector operator.

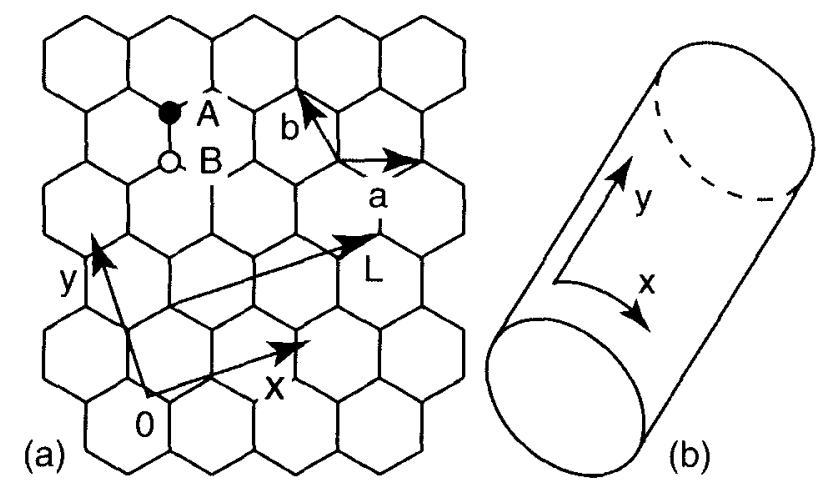

Figure 1. Schematic illustration of (a) 2D graphite and (b) nanotube.

Electronic states for a nanotube with a sufficiently large diameter are obtained by imposing the boundary condition around the circumference direction:

$$
\mathbf{F}(\mathbf{r}+\mathbf{L})=\mathbf{F}(\mathbf{r}) \exp \left(-\frac{2 \pi i \nu}{3}\right)
$$

with $\mathbf{L}$ a chiral vector shown in Fig. 1(a) and $\nu$ an integer determined uniquely as $\nu=0$ (metal) or \pm 1 (semiconductor) through $n_{a}+n_{b}=3 M+\nu$ with integer $M$, where $n_{a}$ and $n_{b}$ are integers defined by $\mathbf{L}=n_{a} \mathbf{a}+n_{b} \mathbf{b}$ and $\mathbf{a}$ and $\mathbf{b}$ are the primitive translation vectors shown in Fig. 1(a). The energy bands are specified by $\alpha=( \pm, n)$ and the wave vector $k$ in the axis direction, where $n$ is an integer, ' - ' denotes the valence band, and ' + ' the conduction band. The wave function for a band associated with the $K$ point is written as

$$
\mathbf{F}_{\alpha k}(x, y)=\frac{1}{\sqrt{A L}} \exp \left[i \kappa_{\nu}(n) x+i k y\right] \mathbf{F}_{\alpha k}^{\nu},
$$

with $A$ being the length of nanotube,

$$
\kappa_{\nu}(n)=\frac{2 \pi}{L}\left(n-\frac{\nu}{3}\right)
$$

and

$$
\mathbf{F}_{\alpha k}^{\nu}=\frac{1}{\sqrt{2}}\left(\begin{array}{c}
b_{\nu}(n, k) \\
s_{\alpha}
\end{array}\right)
$$

where

$$
b_{\nu}(n, k)=\frac{\kappa_{\nu}(n)-i k}{\sqrt{\kappa_{\nu}(n)^{2}+k^{2}}}
$$

and

$$
s_{\alpha}= \begin{cases}+1 & (\alpha=+, n) \\ -1 & (\alpha=-, n)\end{cases}
$$


The corresponding energy is given by

$$
\varepsilon_{ \pm, n}(k)= \pm \gamma \sqrt{\kappa_{\nu}(n)^{2}+k^{2}}
$$

A $\mathbf{k} \cdot \mathbf{p}$ equation near the $K^{\prime}$ point is given by Eq. (1) in which $\sigma_{y}$ is replaced by $\sigma_{y}^{*}$. The wave function and energy are given by Eq. (3) with the complex conjugate of $\mathbf{F}_{\alpha k}^{\nu}$ and Eq. (8), respectively, in which $\nu$ is replaced by $-\nu$.

\subsection{Electron-electron interaction}

The Coulomb potential at $\mathbf{r}=(x, y)$ due to a charge $-e$ at the origin is

$$
V(x, y)=\frac{\pi e^{2}}{\kappa L \sqrt{\sin ^{2}(\pi x / L)+(\pi y / L)^{2}}},
$$

where $\kappa$ is an effective dielectric constant describing screening by electrons in $\sigma$ bands, core states, and the $\pi$ bands away from the $K$ and $K^{\prime}$ points. Then, a matrix element of electron-electron interaction between an initial state specified by single-particle states $(\beta, k)$ and $\left(\alpha^{\prime}, k+q\right)$ and a final state specified by $(\alpha, k+q)$ and $\left(\beta^{\prime}, k\right)$ in a band associated with the $K$ point where $\alpha=( \pm, n), \beta=( \pm, m)$, etc. becomes

$$
\begin{aligned}
v_{(\alpha, k+q ; \beta, k)\left(\beta^{\prime}, k ; \alpha^{\prime}, k+q\right)} & =\int d \mathbf{r}_{1} \int d \mathbf{r}_{2} V\left(\mathbf{r}_{1}-\mathbf{r}_{2}\right)\left[\mathbf{F}_{\alpha k+q}^{*}\left(\mathbf{r}_{1}\right) \cdot \mathbf{F}_{\beta k}\left(\mathbf{r}_{1}\right)\right]\left[\mathbf{F}_{\beta^{\prime} k}^{*}\left(\mathbf{r}_{2}\right) \cdot \mathbf{F}_{\alpha^{\prime} k+q}\left(\mathbf{r}_{2}\right)\right] \\
& =\delta_{n-m, n^{\prime}-m^{\prime}} \frac{2 e^{2}}{\kappa A}\left(\mathbf{F}_{\alpha, k+q}^{\nu *} \cdot \mathbf{F}_{\beta, k}^{\nu}\right)\left(\mathbf{F}_{\beta^{\prime}, k}^{\nu *} \cdot \mathbf{F}_{\alpha^{\prime}, k+q}^{\nu}\right) I_{|n-m|}\left(\frac{L|q|}{2 \pi}\right) K_{|n-m|}\left(\frac{L|q|}{2 \pi}\right),
\end{aligned}
$$

where $I_{n}(t)$ and $K_{n}(t)$ are the modified Bessel functions of the first and second kind of the order $n$, respectively. Around the $K^{\prime}$ point, the matrix element is given by a complex conjugate of Eq. (10) with replacement $\nu \rightarrow-\nu$.

The static polarization function for electrons near the $K$ point calculated in the random-phase approximation becomes

$$
\Pi_{n-m}^{K}(q)=-2 \sum_{\alpha^{\prime}, \beta^{\prime}} \frac{1}{A} \sum_{k^{\prime}} \delta_{n-m, n^{\prime}-m^{\prime}}\left|\mathbf{F}_{\beta^{\prime}, k^{\prime}}^{\nu *} \cdot \mathbf{F}_{\alpha^{\prime}, k^{\prime}+q}^{\nu}\right|^{2} g_{0}\left[\varepsilon_{\alpha^{\prime}}\left(k^{\prime}+q\right)\right] g_{0}\left[\varepsilon_{\beta^{\prime}}\left(k^{\prime}\right)\right] \frac{f_{\alpha^{\prime}, k^{\prime}+q}-f_{\beta^{\prime}, k^{\prime}}}{\varepsilon_{\alpha^{\prime}}\left(k^{\prime}+q\right)-\varepsilon_{\beta^{\prime}}\left(k^{\prime}\right)},
$$

where $f_{\alpha, k}$ is the Fermi-Dirac distribution function for $\varepsilon_{\boldsymbol{\alpha}}(k)$ and $g_{0}(\varepsilon)$ a cutoff function with cutoff energy $\varepsilon_{c}$. defined as

$$
g_{0}(\varepsilon)=\frac{\varepsilon_{c}^{\alpha_{c}}}{|\varepsilon|^{\alpha_{c}}+\varepsilon_{c}^{\alpha_{c}}}
$$

with $\alpha_{c}$ being chosen such that $g_{0}$ decays sufficiently smoothly and the integral converges. The polarization function near the $K^{\prime}$ point, $\Pi_{n-m}^{K^{\prime}}(q)$, is given by Eq. (11) with $\nu \rightarrow-\nu$ and replacement of the energy at the $K$ point $\varepsilon_{\alpha}(k)$ with that at the $K^{\prime}$ point. Then, the dielectric function is given by

$$
\varepsilon_{n-m}(q)=1+\frac{2 e^{2}}{\kappa} I_{|n-m|}\left(\frac{L|q|}{2 \pi}\right) K_{|n-m|}\left(\frac{L|q|}{2 \pi}\right)\left[\Pi_{n-m}^{K}(q)+\Pi_{n-m}^{K^{\prime}}(q)\right] .
$$

The screened Coulomb interaction is given by

$$
V_{(\alpha, k+q ; \beta, k)\left(\beta^{\prime}, k ; \alpha^{\prime}, k+q\right)}=\frac{v_{(\alpha, k+q ; \beta, k)\left(\beta^{\prime}, k ; \alpha^{\prime}, k+q\right)}}{\varepsilon_{n-m}(q)} .
$$

The total Hamiltonian consists of the kinetic term in Eq. (1) and the Coulomb interaction with matrix elements given by Eq. (14).

The cutoff energy should be of the order of the half of the $\pi$-band width $3 \gamma_{0}$, where $\gamma_{0}$ is the resonance integral between nearest neighbor sites and related to the band parameter through $\gamma=(\sqrt{3} / 2) a \gamma_{0}$ with $a=2.46$ $\AA$ being the lattice constant. Therefore, $\varepsilon_{c}(2 \pi \gamma / L)^{-1} \approx(\sqrt{3} / \pi)(L / a)=\sqrt{3} d / a$, with $d$ being the diameter of the 
nanotube. In the following numerical calculations, we use $\varepsilon_{c}(2 \pi \gamma / L)^{-1}=10$ which corresponds to a diameter $\sim 1.4 \mathrm{~nm}$.

The strength of the Coulomb interaction is characterized by the dimensionless parameter $\left(e^{2} / \kappa L\right)(2 \pi \gamma / L)^{-1}$, which is the ratio of the typical Coulomb energy $e^{2} / \kappa L$ and the typical kinetic energy $2 \pi \gamma / L$. For a rough estimate of the interaction strength, we can use the relation $\gamma=(\sqrt{3} / 2) a \gamma_{0}$ with $\gamma_{0} \approx 2.7 \mathrm{eV}$ which well reproduces experimental excitation energies as will be shown in Sec. 3.2 and then have $\left(e^{2} / \kappa L\right)(2 \pi \gamma / L)^{-1} \sim 0.4 / \kappa$. The exact value of $\kappa$ is not known, but we can expect that $\kappa$ is not so much different from 2.4 in bulk graphite. Then, the interaction parameter lies roughly in the range $0.1 \sim 0.2$. Therefore, $\left(e^{2} / \kappa L\right)(2 \pi \gamma / L)^{-1}=0.2$ is used in the following unless specified otherwise.

\subsection{Exciton}

We shall use a screened Hartree-Fock approximation to calculate interaction effect on the band structure and introduce an attractive interaction between a photo-excited electron and a remaining hole using the Coulomb interaction screened by a static dielectric function. Various approximation schemes were compared and this was shown to be sufficient for single-wall nanotubes. ${ }^{33,34}$ Actual calculations can be performed by solving equation of motion for an electron-hole pair. ${ }^{4,5,35}$

An exciton near the $K$ point with the momentum $2 \pi \hbar l / L$ in the circumference direction is written as

$$
|u, l\rangle=\sum_{n, k} \psi_{n}^{l}(k) c_{+, n+l, k}^{\dagger} c_{-, n, k}|g\rangle
$$

where $c_{\alpha, k}^{\dagger}$ and $c_{\alpha, k}$ are the creation and annihilation operators for electrons, respectively, and $|g\rangle$ is the ground state. The equation of ruotim for meriton in the ahove approximation is given by

$$
\left.\varepsilon_{u} \psi_{n}^{l}(k)=\left(\Delta \varepsilon_{n, k}+\Delta \Sigma_{n, k}\right) \psi_{n}, \cdots,-\sum_{m, q} \vdots \quad \ldots, \ldots, m, k+q ;-, n, k\right) \psi_{m}^{l}(k+q),
$$

where

$$
\begin{aligned}
\Delta \varepsilon_{n, k} & =\varepsilon_{+, n+l}(k)-\varepsilon_{-, n}(k), \\
\Delta \Sigma_{n, k} & =\Sigma_{+, n+l, k}-\Sigma_{-, n, k},
\end{aligned}
$$

with $\Sigma_{ \pm, n, k}$ being the self-energy. In the conventional screened Hartree-Fock approximation, it is given by

$$
\Sigma_{ \pm, n, k}^{\prime}=-\sum_{m, q} V_{( \pm, n, k ;-, m, k+q)(-, m, k+q ; \pm, n, k)}
$$

at zero temperature, where the summation is over the occupied valence bands. The particle-hole symmetry is broken in the self-energy of Eq. (19) although very weakly when the cutoff function is introduced. In order to avoid this weak insufficient feature, therefore, we should redefine the self-energy with the particle-hole symmetry as $^{33}$

$$
\Sigma_{ \pm, n, \mathbf{k}}= \pm \frac{1}{2}\left(\Sigma_{+, n, \mathbf{k}}^{\prime}-\Sigma_{-, n, \mathbf{k}}^{\prime}\right)
$$

\subsection{Dynamical conductivity}

Optical absorption is characterized by the dynamical conductivity. In order to calculate it, we use the Kubo formula ${ }^{36}$ where the dynamical conductivity is given by a current-current correlation function. Near the $K$ point the $x$ and $y$ components of the velocity operators for the wave vector $2 \pi l / L$ in the circumference direction are given by

$$
\hat{v}_{\mu}^{l}=\frac{\gamma}{\hbar} \sigma_{\mu} e^{i l \theta}
$$

with $\mu=\{x, y\}$ and $\theta=2 \pi x / L$. At the $K^{\prime}$ point, $\sigma_{\mu}$ is replaced by $\sigma_{\mu}^{*}$. Its matrix element between the ground state and the exciton state is

$$
\left\langle u, l\left|\hat{v}_{\mu}^{l}\right| g\right\rangle=\sum_{n, k}\left(v_{\mu}^{l}\right)_{n, k} \psi_{n}^{l *}(k),
$$


where $\left(v_{\mu}^{l}\right)_{n, k}$ is a matrix element of $\hat{v}_{\mu}^{l}$ between a one-particle state with $\kappa_{\nu}(n+l)$ and $k$ at the conduction band and that with $\kappa_{\nu}(n)$ and $k$ at the valence band for the $K$ point. For the $K^{\prime}$ point it is similarly defined for states with $\kappa_{-\nu}(n+l)$ and $k$ at the conduction band and with $\kappa_{-\nu}(n)$ and $k$ at the valence band. For the $K$ point it becomes

$$
\left(v_{\mu}^{l}\right)_{n, k}^{K}=\frac{\gamma}{\hbar} \mathbf{F}_{+, n+l, k}^{\nu *} \sigma_{\mu} \mathbf{F}_{-, n, k}^{\nu}
$$

and for the $K^{\prime}$ point it is given by complex conjugate of Eq. (23) with $\nu \rightarrow-\nu$.

For light with the momentum $2 \pi \hbar l / L$ in the circumference direction, the associated dynamical conductivity is given by

$$
\sigma_{\mu \mu}^{l}(\omega)=\frac{2 \hbar e^{2}}{A L} \sum_{K K^{\prime}} \sum_{u} \frac{-2 i \hbar \omega\left|\left\langle u, l\left|\hat{v}_{\mu}^{l}\right| g\right\rangle\right|^{2}}{\varepsilon_{u}\left[\varepsilon_{u}^{2}-(\hbar \omega)^{2}-2 i \hbar \omega \Gamma\right]},
$$

where a factor 2 in front of the sum comes from the spin degeneracy, and a phenomenological energy broadening $\Gamma$ has been introduced. For the parallel and perpendicular polarizations, the absorption is characterized by $\sigma_{y y}^{l=0}$ and $\sigma_{x x}^{l= \pm 1}$, respectively. ${ }^{24,25,31}$

For cross-polarized light the depolarization effect must be considered. ${ }^{24,25}$ It is taken into account in a selfconsistent-field method where an optically induced current and electric field which electrons feel are determined self-consistently. ${ }^{24,25}$ Then, absorption power is in proportion to the real part of $\tilde{\sigma}_{x x}(\omega)$ defined as

$$
\tilde{\sigma}_{x x}(\omega)=\frac{1}{2}\left[\tilde{\sigma}_{x x}^{l=1}(\omega)+\tilde{\sigma}_{x x}^{l=-1}(\omega)\right]
$$

where

$$
\tilde{\sigma}_{x x}^{l}(\omega)=\frac{\sigma_{x x}^{l}(\omega)}{\varepsilon_{x x}^{l}(\omega)}
$$

with

$$
\varepsilon_{x x}^{l}(\omega)=1+\frac{4 \pi^{2} i}{\kappa L \omega} \sigma_{x x}^{l}(\omega)
$$

In this case excitation energies are given by zero points $\omega_{0}$ of $\varepsilon_{x x}^{l}(\omega)$ which satisfy

$$
\varepsilon_{x x}^{l}\left(\omega_{0}\right)=1+\frac{4 \pi^{2} i}{\kappa L \omega_{0}} \sigma_{x x}^{l}\left(\omega_{0}\right)=0 .
$$

When the depolarization effect is neglected completely, the absorption is proportional to the real part of the $\sigma_{x x}(\omega)$ given by

$$
\sigma_{x x}(\omega)=\frac{1}{2}\left[\sigma_{x x}^{l=1}(\omega)+\sigma_{x x}^{l=-1}(\omega)\right]
$$

A dimensionless oscillator strength of the lowest exciton absorption peak for parallel polarization is defined as $^{5}$

$$
f_{u}=2 m^{*} \frac{\left|\left\langle u\left|\hat{v}_{y}\right| g\right\rangle\right|^{2}}{\varepsilon_{u}}
$$

where with $|u\rangle=|u, l=0\rangle$ in Eq. (15), $\hat{v}_{y}=\hat{v}_{y}^{l=0}$ in Eq. (21), and the effective mass of the first conduction band is defined by

$$
m^{*}=\frac{2 \pi \hbar^{2}}{3 \gamma L}
$$

The oscillator strength for perpendicular polarization is given in the same way by

$$
f_{u}=2 m^{*} \frac{\kappa L^{3} \omega_{0}}{4 \pi^{2} \hbar e^{2}}\left[\frac{1}{\hbar} \frac{\partial \varepsilon_{x x}^{l}}{\partial \omega}\left(\omega_{0}\right)\right]^{-1} .
$$




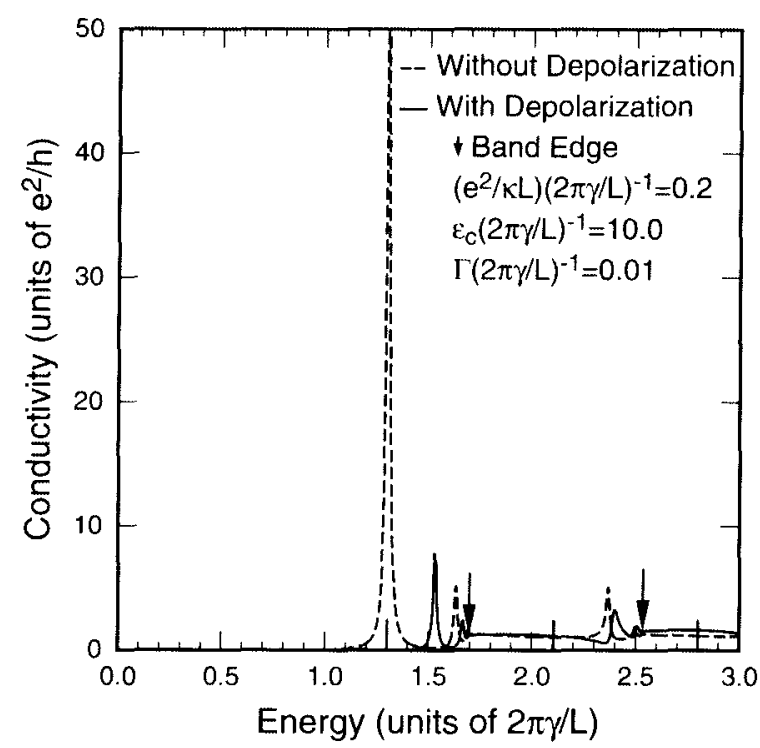

Figure 2. Dynamical conductivity for cross-polarized light in a semiconducting tube for a phenomenological energy broadening $\Gamma(2 \pi \gamma / L)^{-1}=0.01$. Solid and dashed lines denote results with and without the depolarization effect, respectively.

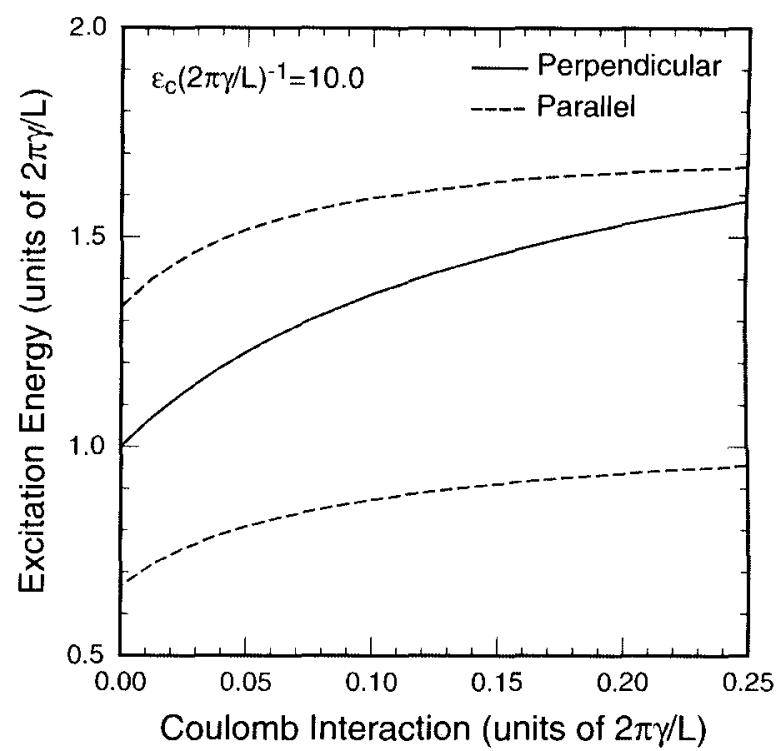

Figure 3. Coulomb-interaction dependence of excitation energies for perpendicular (solid line) and parallel (dashed lines) polarization.

\subsection{Two-photon absorption}

In calculations of two-photon absorption, we shall confine ourselves to the low-energy regime below the interband continuum and therefore completely neglect multi-exciton states such as a bi-exciton. For parallel polarization where the electric field is given by

$$
E_{x}=0, \quad E_{y}=E\left(e^{-i \omega t}+e^{i \omega t}\right),
$$

a two-photon energy-absorption rate per unit length is given by $\beta(\omega) E^{4}$ with an absorption coefficient

$$
\beta(\omega)=\frac{4 \pi e^{4}}{\omega^{3} A} \sum_{K, K^{\prime}} \sum_{u}\left|\sum_{u^{\prime}} \frac{\left\langle u\left|\hat{v}_{y}\right| u^{\prime}\right\rangle\left\langle u^{\prime}\left|\hat{v}_{y}\right| g\right\rangle}{\hbar \omega-\varepsilon_{u^{\prime}}}\right|^{2} \delta\left(2 \hbar \omega-\varepsilon_{u}\right) .
$$

Using typical scales of physical quantities in nanotubes, a dimensionless absorption coefficient $\bar{\beta}(\omega)$ is given by

$$
\beta(\omega)=\frac{e^{4} L^{5}}{8 \pi^{3} \hbar \gamma^{2}} \bar{\beta}(\omega)
$$

Then, we can see that the absorption coefficient is proportional to the fifth power of the circumference length.

The velocity matrix elements for the $K$ point are given by

$$
\left\langle u^{\prime}\left|\hat{v}_{y}\right| g\right\rangle=\frac{i \gamma}{\hbar} \sum_{n} \sum_{k} \frac{\kappa_{\nu}(n)}{\sqrt{\kappa_{\nu}(n)^{2}+k^{2}}} \psi_{n}^{u^{\prime *}}(k),
$$

and

$$
\left\langle u\left|\hat{v}_{y}\right| u^{\prime}\right\rangle=-\frac{2 \gamma}{\hbar} \sum_{n} \sum_{k} \frac{k}{\sqrt{\kappa_{\nu}(n)^{2}+k^{2}}} \psi_{n}^{u *}(k) \psi_{n}^{u^{\prime}}(k),
$$

where $\psi_{n}^{u}(k)$ is the wave function $\psi_{n}^{l}(k)$ in Eq. (15) for an exciton denoted by $u$ with the zero momentum $l=0$. Those for the $K^{\prime}$ point are given by the complex conjugate of the above with the replacement $\nu \rightarrow-\nu$. The wave function $\psi_{n}^{u}(k)$ is an even or odd function of $k$, that is,

$$
\psi_{n}^{u}(-k)= \pm \psi_{n}^{u}(k)
$$




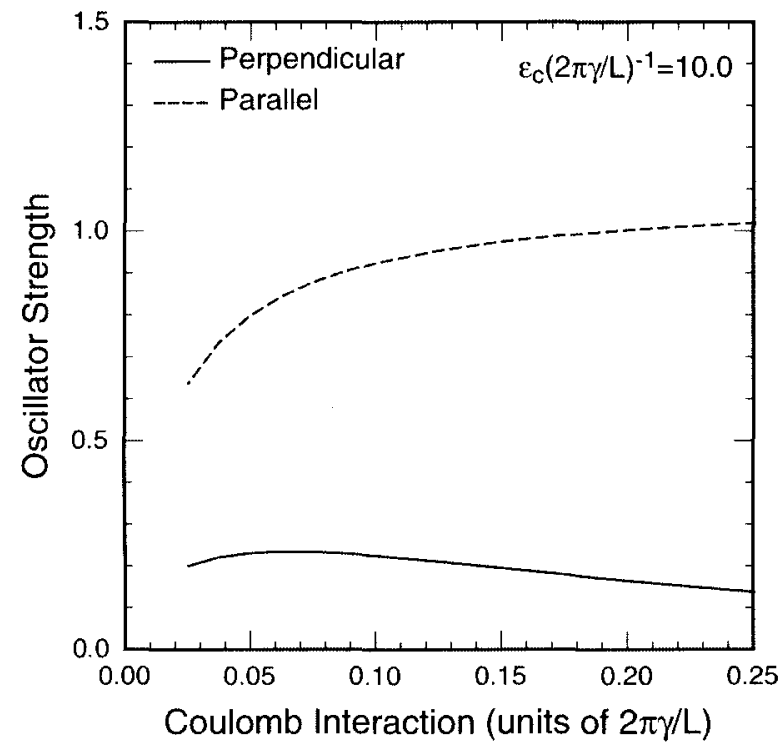

Figure 4. Coulomb-interaction dependence of oscillator strength for perpendicular (solid line) and parallel (dashed line) polarization.

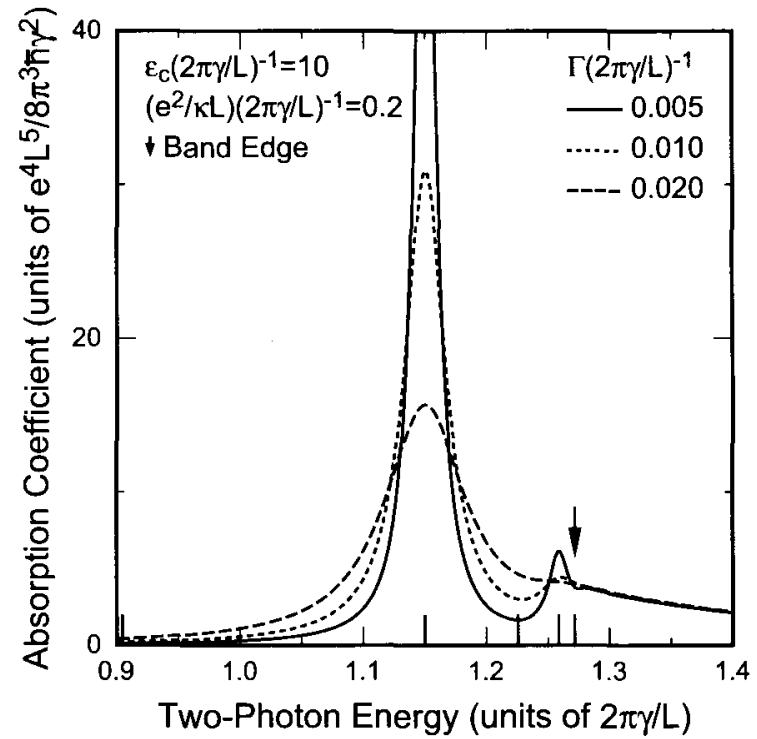

Figure 5. Two-photon absorption coefficient for parallel polarization. Phenomenological broadening $\Gamma(2 \pi \gamma / L)^{-1}$ is introduced.

Therefore, it can be seen from Eqs. (36) and (37) that states with even parity are excited from the ground state by one-photon absorption and those with odd parity are excited by two-photon absorption. This selection rule obtained within the effective-mass scheme corresponds to the more general rule based on the symmetry under the $\pi$ rotation around the center of a carbon-atom hexagon obtained previously. ${ }^{21,23,37}$

\section{NUMERICAL RESULTS}

\subsection{Cross-polarized light}

Figure 2 shows the dynamical conductivity for light polarized perpendicular to a semiconducting tube. The solid and dashed lines show conductivity with and without the depolarization effect, respectively. The vertical arrows indicate band edges. When the depolarization effect is not considered, the largest peak appears below the lowest band edge. When the depolarization effect is taken into account, the peak is shifted toward the higher energy side below the band edge and its intensity is reduced.

In Fig. 3 solid and dashed lines show the Coulomb-interaction dependence of the excitation energy for perpendicular polarization and those for parallel polarization associated with the first and second lowest subbands, respectively. In the absence of interaction, the lowest band edge for the perpendicular polarization is located at the middle of lowest two bands for the parallel polarization. Because the interaction dependence of the energy for the perpendicular light is stronger than that for the parallel light due to the depolarization effect, the energy for the perpendicular polarization becomes close to the second gap for the parallel polarization with increase of the interaction.

Solid and dashed lines in Fig. 4 show the oscillator strength of the lowest exciton absorption peaks for the perpendicular and parallel polarization, respectively, as a function of the Coulomb interaction. The oscillator strength for the perpendicular polarization lies typically in the range from ten to thirty percent of that for the parallel polarization.

\subsection{Two-photon absorption}

Figure 5 shows typical examples of energy dependence of two-photon absorption coefficient for parallel polarization in semiconducting tubes where energy broadening is introduced by using a Lorentzian function with a 


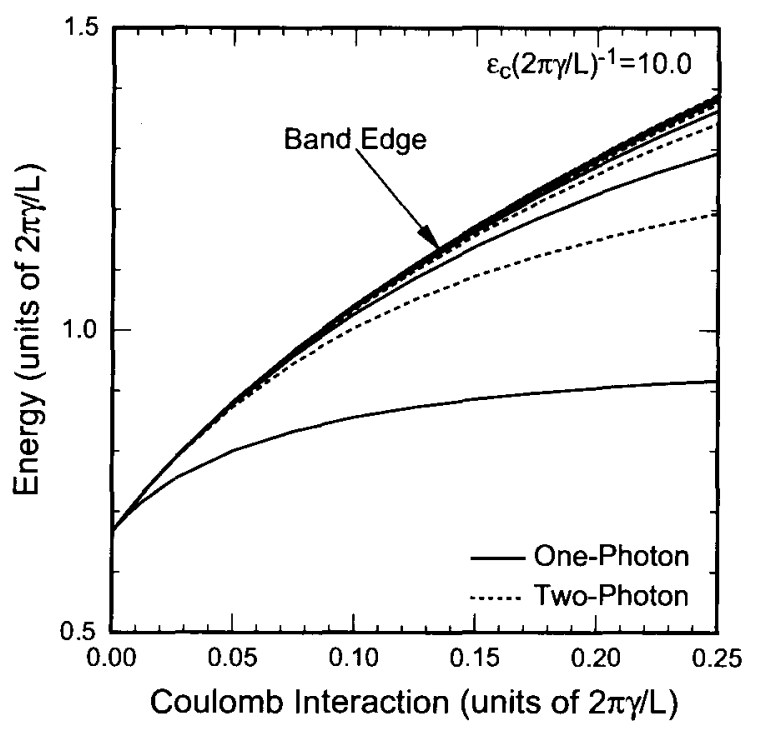

Figure 6. Coulomb-interaction dependence of exciton energies associated with the lowest band gap. Solid and dotted lines denote excitons excited by one- and two-photon absorption, respectively.

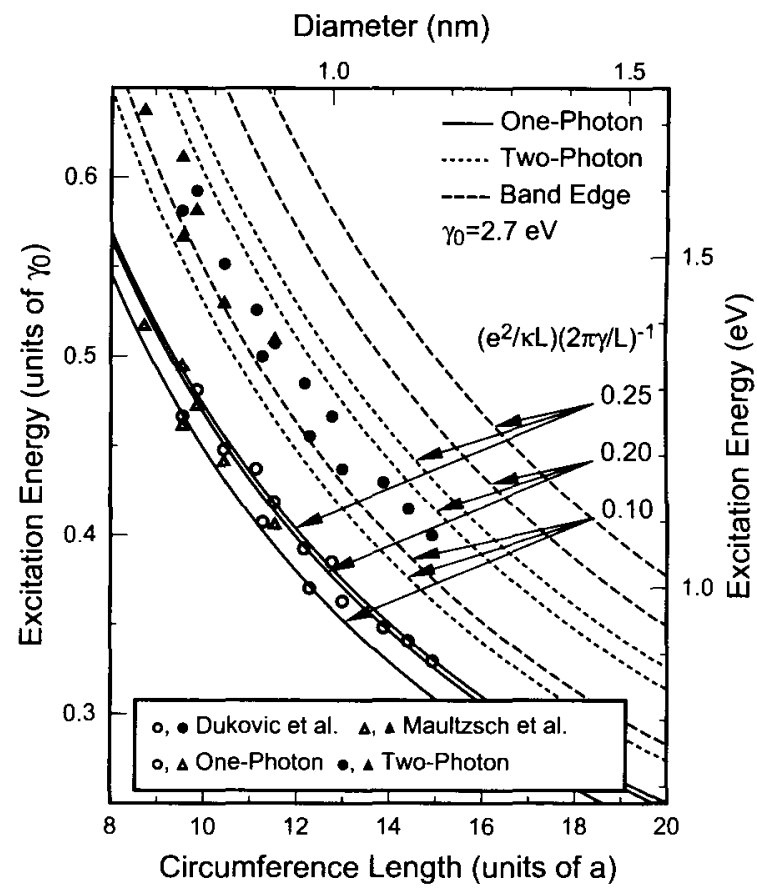

Figure 7. Calculated excitation energies as a function of the circumference length. Solid and dotted lines denote the energies for one- and two-photon absorption, respectively, and dashed lines are the band edges. Symbols denote experimental results ${ }^{21,22}$ where open and closed symbols indicate one- and two-photon transitions, respectively.

half width at half maximum $\Gamma$. The energies of all the exciton bound states and the lowest band edge are indicated by short vertical lines at the bottom and by a downward arrow, respectively. A prominent peak appears corresponding to the second lowest exciton with odd parity and another peak associated with the fourth lowest exciton appears. The difference between the first and second lowest exciton energies is approximately 0.22 in units of $2 \pi \gamma / L$, which is about $0.18 \mathrm{eV}$ for $\gamma_{0}=2.7 \mathrm{eV}$.

In Fig. 6, the Coulomb-interaction dependence of the exciton energies is shown. Solid and dotted lines denote excitons contributing to one- and two-photon absorption spectra, respectively. With increase of the interaction, many exciton bound states split off from the interband continuum. Similar results were previously reported for a different cutoff energy. ${ }^{4}$

In Fig. 7, excitation energies are plotted as a function of the circumference length. Solid and dotted lines denote calculated energies of the two lowest excitons observable in one- and two-photon absorption, respectively, and dashed lines indicate band edges. Symbols are experimental results ${ }^{21,22}$ where open and filled symbols indicate excitons for one- and two-photon transitions, respectively. We have set $\gamma_{0} \approx 2.7 \mathrm{eV}$ which was used for comparison with experiments for the lowest excitons associated with the first and second gaps in a previous paper. ${ }^{5}$

As shown in Fig. 6, the energy of the lowest exciton is weakly dependent on the interaction strength $\left(e^{2} / \kappa L\right)(2 \pi \gamma / L)^{-1}$. Therefore, the band parameter is uniquely determined as approximately $\gamma_{0} \approx 2.7 \mathrm{eV}$. Using the position of the second exciton, strongly dependent on the Coulomb interaction, we can place the interaction parameter in the range $0.1<\left(e^{2} / \kappa L\right)(2 \pi \gamma / L)^{-1}<0.2$. For $d \sim 1 \mathrm{~nm}$, for example, the binding energy of the lowest exciton changes from $\sim 0.21$ to $\sim 0.43 \mathrm{eV}$ in this interaction range. 


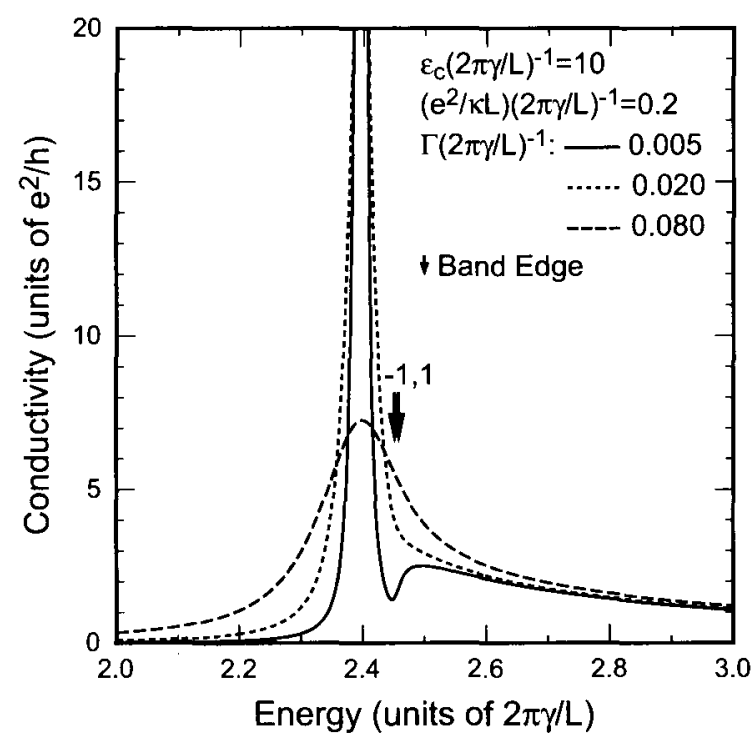

Figure 8. Dynamical conductivity for parallel polarization in a metallic tube.

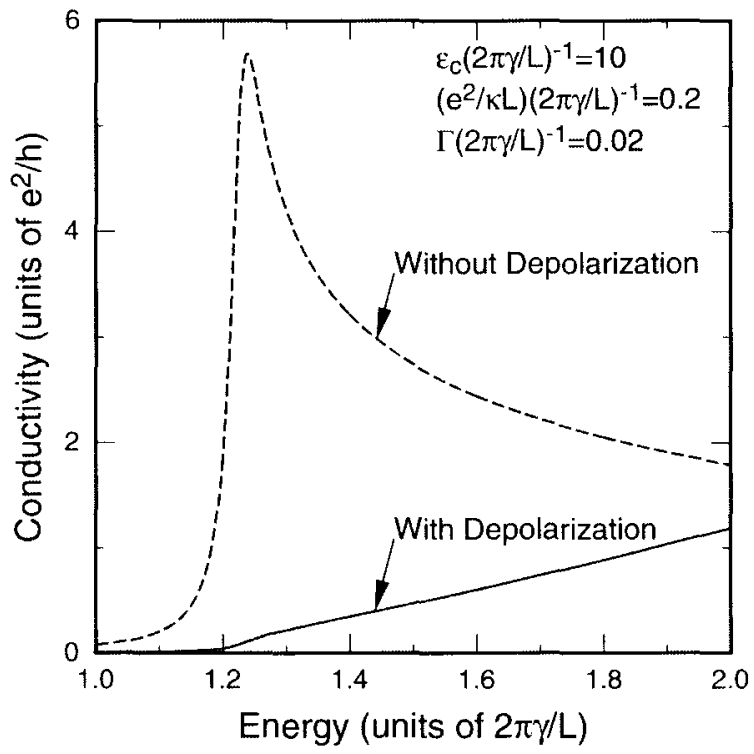

Figure 9. Calculated dynamical conductivity for perpendicular polarization in a metallic tube. An energy broadening $\Gamma(2 \pi \gamma / L)^{-1}=0.02$ is used. Solid and dashed lines denote results with and without the depolarization effect, respectively.

\subsection{Metallic tube}

In this subsection, metallic tubes are considered. In Fig. 8, typical optical absorption spectra for parallel polarization are shown in an energy region corresponding to transition between the bands with $n= \pm 1$ below and above the linear dispersions. At around energy $2.4 \times(2 \pi \gamma / L)$, a sharp exciton peak appears below the band edge denoted by arrows. In the energy region higher than the band edge, contributions from continuum states can be seen as a small and long tail. The typical exciton binding energy is about 0.05 in units of $2 \pi \gamma / L$.

Figure 9 shows typical absorption spectra for perpendicular polarization. Solid and dashed lines are the results with and without the depolarization effect, respectively. The conductivity without the depolarization effect shows a peak and it disappears when the depolarization effect is considered. This is quite in contrast to the case of semiconducting tubes shown in Fig. 2.

A potential with a range larger than the lattice constant, including that of the Coulomb interaction, cannot cause backward scattering within the linear bands. ${ }^{38,39}$ Either of an electron or a hole of the electron-hole pair is located at one of the two linear bands for the perpendicular polarization. ${ }^{24,25}$ Because it cannot be scattered between the positive and negative wave-vector region by the Coulomb interaction, the exciton consists of an electron-hole pair with the same sign of wave vectors. This leads to the extremely weak binding of the exciton for the perpendicular polarization.

\section{DISCUSSIONS}

As shown in Fig. 3 the Coulomb-interaction dependence of the exciton energy for perpendicular polarization is stronger than that for parallel polarization. This can be used to estimate the interaction strength from experiments for cross-polarized-light absorption. In experiments using tubes isolated with aromatic polymers, ${ }^{29}$ the Coulomb interaction strength was estimated to be from 0.122 to $0.155 \mathrm{eV}$ for $\gamma_{0}=2.75 \mathrm{eV}$ depending on tube structure, which is converted to $0.1 \lesssim\left(e^{2} / \kappa L\right)(2 \pi \gamma / L)^{-1} \lesssim 0.12$ by considering tube diameters. It should be noted that since the interaction strength is sensitive to surrounding materials, it is expected to become larger for tubes suspended in air. 
For two metallic $(12,0)$ and $(10,10)$ tubes subjected to light polarized parallel to the tube axis, first-principles calculations gave about $50 \mathrm{meV}$ to the exciton binding energy of $n= \pm 1$ transitions. ${ }^{15}$ Our calculated value $0.05 \times(2 \pi \gamma / L)$ for both $\left(e^{2} / \kappa L\right)(2 \pi \gamma / L)^{-1}=0.1$ (not shown here) and 0.2 approximately gives $60 \mathrm{meV}$ in a $(12,0)$ tube and $40 \mathrm{meV}$ in a $(10,10)$ tube for $\gamma_{0}=2.7 \mathrm{eV}$. It can be said at least that these results are consistent with each other.

Measured excitation energies exhibit chiral-angle dependence called family patterns. They are not considered in our calculations because they are described by higher order corrections in the effective mass approximation which correspond to trigonal warping, curvature, and lattice distortions. ${ }^{5,40}$ Recent calculations for parallel polarization showed that inclusion of these corrections well reproduces experimental one- and two-photon excitation energies with the family patterns. ${ }^{40}$ Similar calculations for perpendicular polarization are expected to give information on a parameter describing electron-hole asymmetry, which has negligible effects on absorption for parallel polarization. This remains as a future issue.

\section{SUMMARY AND CONCLUSION}

Exciton effects in carbon nanotubes have been theoretically studied in an effective-mass approximation. For crosspolarized-light absorption in semiconducting tubes, distinct exciton peaks appear in spite of the depolarization effect although their intensity is reduced as compared to that for parallel polarization. Calculated excited exciton energies are in good agreement with those obtained by two-photon absorption experiments. An exciton can exist in metallic tubes also.

\section{Acknowledgments}

This work was supported in part by a Global Center of Excellence Program at Tokyo Tech "Nanoscience and Quantum Physics," Grants-in-Aid for Scientific Research, and Grant-in-Aid for Scientific Research on Priority Area "Carbon Nanotube Nanoelectronics" from the Ministry of Education, Culture, Sports, Science and Technology, Japan.

\section{REFERENCES}

[1] Uryu, S. and Ando, T., "Exciton absorption of perpendicularly polarized light in carbon nanotubes," Phys. Rev. B 74, 155411-1-9 (2006).

[2] Uryu, S. and Ando, T., "Excitons in metallic carbon nanotubes with Aharonov-Bohm flux," Phys. Rev. B 77, 205407-1-9 (2008).

[3] Uryu, S. and Ando, T., "Excitonic two-photon absorption in semiconducting carbon nanotubes within an effective-mass approximation," Phys. Rev. B 78, 115414-1-6 (2008).

[4] Ando, T., "Excitons in carbon nanotubes," J. Phys. Soc. Jpn. 66, 1066-1073 (1997).

[5] Ando, T., "Excitons in carbon nanotubes revisited: Dependence on diameter, Aharonov-Bohm flux, and strain," J. Phys. Soc. Jpn. 73, 3351 3363 (2004).

[6] Spataru, C. D., Ismail-Beigi, S., Benedict, L. X., and Louie, S. G., "Excitonic effects and optical spectra of single-walled carbon nanotubes ," Phys. Rev. Lett. 92, 077402-1-4 (2004).

[7] Chang, E., Bussi, G., Ruini, A., and Molinari, E., "Excitons in carbon nanotubes: An ab initio symmetrybased approach," Phys. Rev. Lett. 92, 196401-1-4 (2004).

[8] Perebeinos, V., Tersoff, J., and Avouris, Ph., "Scaling of excitons in carbon nanotubes," Phys. Rev. Lett. 92, 257402-1-4 (2004).

[9] Zhao, H. and Mazumdar, S., "Electron-electron interaction effects on the optical excitations of semiconducting single-walled carbon nanotubes," Phys. Rev. Lett. 93, 157402-1-4 (2004).

[10] Kane, C. L. and Mele, E. J., "Electron interactions and scaling relations for optical excitations in carbon nanotubes," Phys. Rev. Lett. 93, 197402-1-4 (2004).

[11] Ichida, M., Mizuno, S., Tani, Y., Saito, Y., and Nakamura, A., "Exciton effects of optical transitions in single-wall carbon nanotubes," J. Phys. Soc. Jpn. 68, 3131-3133 (1999). 
[12] O'Connell, M. J., Bachilo, S. M., Huffman, C. B., Moore, V. C., Strano, M. S., Haroz, E. H., Rialon, K. L., Boul, P. J., Noon, W. H., Kittrell, C., Ma, J., Hauge, R. H., Weisman, R. B., and Smalley, R. E., "Band gap fluorescence from individual single-walled carbon nanotubes," Science 297, 593-596 (2002).

[13] Bachilo, S. M., Strano, M. S., Kittrell, C., Hauge, R. H., Smalley, R. E., and Weisman, R. B., "Structureassigned optical spectra of single-walled carbon nanotubes," Science 298, 2361-2366 (2002).

[14] Wang, F., Cho, D. J., Kessler, B., Deslippe, J., Schuck, P. J., Louie, S. G., Zettl, A., Heinz, T. F., and Shen, Y. R., "Observation of excitons in one-dimensional metallic single-walled carbon nanotubes," Phys. Rev. Lett. 99, 227401-1-4 (2007).

[15] Deslippe, J., Spataru, C. D., Prendergast, D., and Louie, S. G., "Bound excitons in metallic single-walled carbon nanotubes," Nano Lett. 7, 1626-1630 (2007).

[16] Margulis, Vl. A. and Sizikova, T. A., "Theoretical study of third-order nonlinear optical response of semiconductor carbon nanotubes," Physica B 245, 173-189 (1998).

[17] Margulis, Vl. A. and Gaiduk, E. A., "Theoretical modeling of nonlinear refraction and two-photon absorption in single-wall carbon nanotube bundles," J. Opt. A: Pure Appl. Opt. 3, 267-275 (2001).

[18] Mele, E. J., Kral, P., and Tomanek, D., "Coherent control of photocurrents in graphene and carbon nanotubes," Phys. Rev. B 61, 7669-7677 (2000).

[19] Cheng, W.-D., Wu, D.-S., Li, X.-D., Lan, Y.-Z., Zhang, H., Chen, D.-G., Gong, Y.-J., Zhang, Y.-C., Li, F.F., Shen, J., and Kan, Z.-G., "Design of single-walled carbon nanotubes with a large two-photon absorption cross section," Phys. Rev. B 70, 155401-1-6 (2004).

[20] Wang, F., Dukovic, G., Brus, L. E., and Heinz, T. F., "The optical resonances in carbon nanotubes arise from excitons," Science 308, 838-841 (2005).

[21] Maultzsch, J., Pomraenke, R., Reich, S., Chang, E., Prezzi, D., Ruini, A., Molinari, E., Strano, M. S., Thomsen, C., and Lienau, C., "Exciton binding energies in carbon nanotubes from two-photon photoluminescence," Phys. Rev. B 72, 241402-1-4(R) (2005).

[22] Dukovic, G., Wang, F., Song, D., Sfeir, M. Y., Heinz, T. F., and Brus, L. E., "Structural dependence of excitonic optical transitions and band-gap energies in carbon nanotubes," Nano Lett. 5, 2314-2318 (2005).

[23] Zhao, H., Mazumdar, S., Sheng, C.-X., Tong, M., and Vardeny, Z. V., "Photophysics of excitons in quasi-onedimensional organic semiconductors: Single-walled carbon nanotubes and $\pi$-conjugated polymers," Phys. Rev. B 73, 075403-1-11 (2006).

[24] Ajiki,H. and Ando, T., "Aharonov-Bohm effect in carbon nanotubes," Physica B 201, 349-352 (1994).

[25] Ajiki, H. and Ando, T., "Carbon nanotubes: Optical absorption in Aharonov-Bohm flux," Jpn. J. Appl. Phys. Suppl. 34-1, 107-109 (1995).

[26] Uryu,S. and Ando, T., "Cross polarized absorption in carbon nanotubes with Aharonov-Bohm flux," Phys. Rev. B 76, 115420-1-6 (2007).

[27] Miyauchi, Y., Oba, M., and Maruyama, S., "Cross-polarized optical absorption of single-walled nanotubes probed by polarized photoluminescence excitation spectroscopy," Phys. Rev. B 74, 205440-1-6 (2006).

[28] Lefebvre,J. and Finnie, P., "Polarized photoluminescence excitation spectroscopy of single-walled carbon nanotubes," Phys. Rev. Lett. 98, 167406-1-4 (2007).

[29] Chuang, K.-C., Nish, A., Hwang, J.-Y., Evans, G. W., and Nicholas, R. J., "Experimental study of Coulomb corrections and single-particle energies for single-walled carbon nanotubes using cross-polarized photoluminescence," Phys. Rev. B 78, 085411-1-5 (2008).

[30] Ajiki, H. and Ando, T., "Electronic states of carbon nanotubes," J. Phys. Soc. Jpn. 62, 1255-1266 (1993).

[31] Ando, T., "Theory of electronic states and transport in carbon nanotubes," J. Phys. Soc. Jpn. 74, 777-817 (2005).

[32] Slonczewski, J. C. and Weiss, P. R., "Band structure of graphite," Phys. Rev. 109, 272-279 (1958).

[33] Sakai, H., Suzuura, H., and Ando, T., "Effective-mass theory of electron correlations in band structure of semiconducting carbon nanotubes," J. Phys. Soc. Jpn. 72, 1698-1705 (2003).

[34] Sakai, H., Suzuura, H., and Ando, T., "Effective-mass approach to interaction effects on electronic structure in carbon nanotubes," Physica E 22, 704-707 (2004).

[35] Ando, T., "Effects of valley mixing and exchange on excitons in carbon nanotubes with Aharonov-Bohm flux," J. Phys. Soc. Jpn. 75, 024707-1-12 (2006). 
[36] Kubo, R., "Statistical-mechanical theory of irreversible processes. I. General theory and simple applications to magnetic and conduction problems," J. Phys. Soc. Jpn. 12, 570-586 (1957).

[37] Damnjanović, M., Milošević, I., Vuković, T., and Sredanović, R., "Full symmetry, optical activity, and potentials of single-wall and multiwall nanotubes," Phys. Rev. B 60, 2728-2739 (1999).

[38] Ando, T. and Nakanishi, T., "Impurity scattering in carbon nanotubes - absence of back scattering -," $J$. Phys. Soc. Jpn. 67, 1704-1713 (1998).

[39] Ando,T., Nakanishi, T., and Saito, R., "Berry's phase and absence of back scattering in carbon nanotubes," J. Phys. Soc. Jpn. 67, 2857-2862 (1998).

[40] Ando, T., "Family effects on excitons in semiconducting carbon nanotubes," J. Phys. Soc. Jpn. 78, 1047031-9 (2009). 